\title{
Correction to: Polarisation non-reciprocity cancelling in Sagnac fibre ring interferometer: an attempt of realistic study
}

\author{
Alexander M. Kurbatov ${ }^{1} \cdot$ Roman A. Kurbatov ${ }^{1}$
}

Published online: 3 November 2021

(c) Springer Science+Business Media, LLC, part of Springer Nature 2021

\section{Correction to: Optical and Quantum Electronics (2019) 51:142 https://doi.org/10.1007/s11082-019-1851-7}

After publication of the paper, authors realised that there is a problem with Jones matrix of the input polarising (PZ) lightguide. It yielded many correct results for fibres and for simplified configurations of Sagnac fibre ring interferometer (FRI) studied earlier analytically. However, it was then found that it does not yield the same results for uniformly twisted PZlightguide within Sagnac FRI and for the same twisted PZ-lightguide within FRI being represented as divided into several pieces of the same summary length. This could cause the errors in the final result of the paper, which is very optimistic, despite of one (surmountable) limitation due to PZ-lightguide uniform twist. Fortunately, correct Jones matrix leads to even more optimistic predictions with no such limitation. Below are the corrections.

1. Correction to Sect. 5.5. Starting from the last sentence of the second paragraph ("As a result..."), the rest of Sect. 5.5 should look like the following. As a result, Jones matrix for PZ-lightguide of the length $l$ is yielded in the form

$$
P Z=\left[\begin{array}{cc}
C-i(\Delta b / \mathrm{B}) S & 2(\Theta / \mathrm{B}) S \\
-2(\Theta / \mathrm{B}) S & C+i(\Delta b / \mathrm{B}) S
\end{array}\right],
$$

where $C \equiv\left(e_{+}+e_{-}\right) / 2, S \equiv\left(e_{+}-e_{-}\right) /(2 i), e_{+} \equiv \exp \left(\mathrm{T}_{+}\right), \mathrm{T}_{ \pm} \equiv\left( \pm i \mathrm{~B}-\xi_{\text {in }} / 2\right) l / 2$, $\mathrm{B} \equiv \sqrt{\Delta b^{2}+4 \Theta^{2}}, \Delta b \equiv \Delta \beta+i \xi_{\text {in }} / 2\left(\Delta \beta \equiv \beta_{x}-\beta_{y}\right.$ is propagation constants difference of polarisation $x$ - and $y$-modes, $\xi_{i n}=\xi_{i n, y}-\xi_{i n, x}$ is losses difference of $y$ - and $x$-mode). This model is used for simulation of PZ-lightguide with the sum of uniform and random twists; this sum is also assumed for a coil fibre and coupler fibre leads. Random twists and lengths are simulated by random numbers generators. Finally, attenuation of $y$-mode is $30 \mathrm{~dB} / \mathrm{m}$.

For this kind of Jones matrix for PZ-lightguide, "interferometric" polarisation extinction ratio (PER), introduced in Sect. 3.2 and in Appendix 1, is calculated as a determi-

The original article can be found online at https://doi.org/10.1007/s11082-019-1851-7.

Roman A. Kurbatov

romuald75@mail.ru

1 Kuznetsov Research Institute for Applied Mechanics, Aviamotornaya St., 55, Moscow, Russia 111123 
nant of this matrix PZ (Appendix 1). For uniformly twisted input PZ-lightguide, it is simply equal to.

$\operatorname{det} P Z=\exp \left(\xi_{\text {in }} L_{\text {in }} / 2\right)$,

being, thus, completely independent on the lightguide twist $\Theta$. As for PZ-lightguide with random twists, its determinant has the same above value, because.

$\operatorname{det} P Z=\prod_{n=1}^{N} \exp \left(\xi_{\text {in }} l_{n} / 2\right)=\exp \left(\xi_{\text {in }} \sum_{n=1}^{N} l_{n} / 2\right)=\exp \left(\xi_{\text {in }} L_{\text {in }} / 2\right)$.

This is also the case for the sum of uniform and random twists.

2. According to the above, Sect. 6 and 7 in their complete forms should look like the following.

\section{PNR simulation for quasi-minimal FRI}

Figure 2 (left) shows the graphs for $P N R_{1-3}$ (right part of the Fig. 2 is related to the following Sect. 7). Coil fibre parameters are the following: $B=3 \times 10^{-4}, h=7 \times 10^{-6} 1 / \mathrm{m}$, $2 R=50 \mathrm{~mm}, L=100 \mathrm{~m}, B_{\text {in }}=10 \times 10^{-4}, P E R_{I O C}=50 \mathrm{~dB}$ (small-size medium-quality FRI). Approximation by $G$-value from Eq. (4) occurs for $P N R_{2,3}$, in analogy with $\varepsilon^{1}$ -dependence in Eqs. (1), (3) and (4). Also, approximation by $G^{2}$ occurs for $P N R_{1}$, in analogy with $\varepsilon^{2}$-dependence in Eqs. (1), (3) and (4), but only for $L_{i n}<0.8 \mathrm{~m}$; for $L_{i n}>0.8 \mathrm{~m}$, approximation becomes of the form $G^{1}$. Because all three required $P N R_{1-3} \sim 10^{-10} \mathrm{deg} / \mathrm{h}$ are reached at $L_{i n}>0.8 \mathrm{~m}$ one may write $P N R_{1-3} \sim G$. This means PNR cancelling in agreement with analytical approach for lowest-order PMC described in "Appendix 1". Therefore, "interferometric" PER of PZ-lightguide is described by Eq. (5), being proportional to $L_{i n}$, while the "intensity" PER is the same $(60 \mathrm{~dB})$ for all $L_{i n}>1 / \xi_{i n} \sim 1 \mathrm{~m}$.

Also, total "interferometric" PER of PZ-lightguide and IOC guides is just the sum of their own "interferometric" PER values (Sect. 3.2 and "Appendix 1"), so IOC guides may be non-polarising, because for the same result, only 2-m longer input PZ-lightguide is enough.

\section{PNR simulation for complete FRI with the coupler of low-birefringent (SM) fibre}

Carrara et al. (1987) described $P N R_{1}$ growth due to the fibre coupler. Figure 2 (right) contains $P N R_{1,2,3}$ graphs for the case of the coupler manufactured of low-birefringent (SM) fibre (FRI parameters are the same as in Sect. 6). For $P N R_{0}$, which in not a priori zero, new calculations, nevertheless, yield values below $10^{-11} \mathrm{deg} / \mathrm{h}$ for all $L_{i n}$, so this graph is not shown at Fig. 2. In this case of complete FRI, angle between the axes of
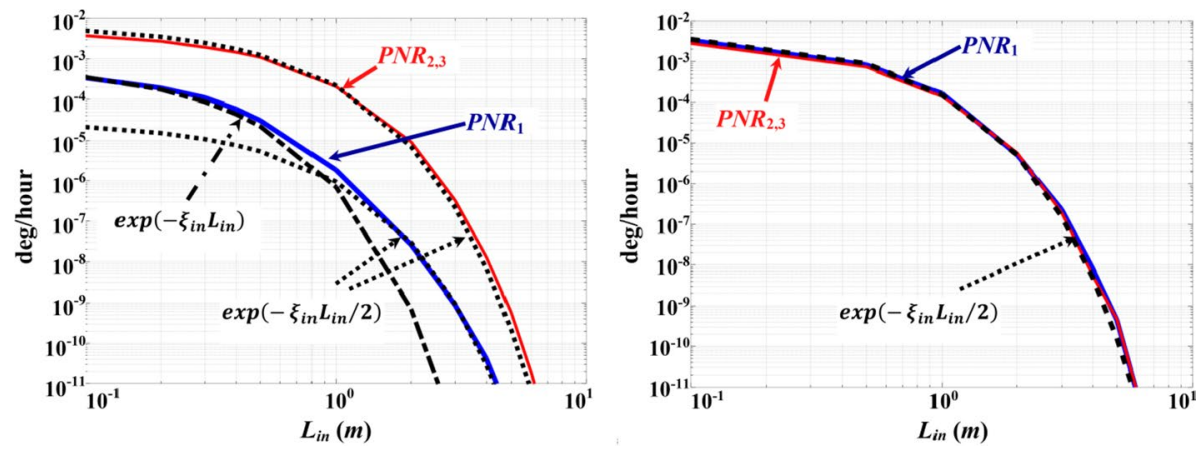

Fig. 2 PNR dependence on the input PZ-lightguide length $L_{i n}$ for quasi-minimal FRI (left) and complete FRI with the coupler of low-birefringent (SM) fibre 
SM-fibre lead "12" and PZ-lightguide is set to be 45 degrees; this mimics the situation when it is out of control during their splicing so some average value has been chosen.

Only $P N R_{1}$ is grown comparing to Fig. 2 (left), which is, at least, in formal agreement with Carrara et al. (1987), despite of the fact that they did not treat decoherence effects.

Thus, less than 10-m PZ-lightguide is able to cancel the polarisation non-reciprocity of Sagnac FRI without any limitations which could arise due to its twists (random, uniform, or their sum). This occurs despite of the small standard ("intensity") PER value (40-60 dB) of the twisted PZ-lightguide because of the suitable form of PZ-lightguide Jones matrix elements; the latter physically means that spurious waves, generated under the influence of PZ-lightguide twists, are the same for cw- and ccw-waves of Sagnac FRI (no PNR).

3. Sixth paragraph of Appendix 1 should be added by the sentence: "But there is no need for this, because, "interferometric" PER of PZ-lightguide does not depend on its twist, according to its Jones matrix (Sect. 5.5)".

Publisher's Note Springer Nature remains neutral with regard to jurisdictional claims in published maps and institutional affiliations. 